\title{
Dissection of molecular mechanisms underlying speech and language disorders
}

\author{
SIMON E. FISHER \\ Wellcome Trust Centre for Human Genetics
}

\section{ADDRESS FOR CORRESPONDENCE}

Simon E. Fisher, Wellcome Trust Centre for Human Genetics, Roosevelt Drive, Oxford OX3 7BN, UK. E-mail: simon.fisher@well.ox.ac.uk

\begin{abstract}
Developmental disorders affecting speech and language are highly heritable, but very little is currently understood about the neuromolecular mechanisms that underlie these traits. Integration of data from diverse research areas, including linguistics, neuropsychology, neuroimaging, genetics, molecular neuroscience, developmental biology, and evolutionary anthropology, is becoming essential for unraveling the relevant pathways. Recent studies of the FOXP2 gene provide a case in point. Mutation of FOXP2 causes a rare form of speech and language disorder, and the gene appears to be a crucial regulator of embryonic development for several tissues. Molecular investigations of the central nervous system indicate that the gene may be involved in establishing and maintaining connectivity of corticostriatal and olivocerebellar circuits in mammals. Notably, it has been shown that FOXP2 was subject to positive selection in recent human evolution. Consideration of findings from multiple levels of analysis demonstrates that FOXP2 cannot be characterized as "the gene for speech," but rather as one critical piece of a complex puzzle. This story gives a flavor of what is to come in this field and indicates that anyone expecting simple explanations of etiology or evolution should be prepared for some intriguing surprises.
\end{abstract}

It has been clearly demonstrated in the past two decades or so that developmental disorders of speech and language have a strong inherited component (Bishop, 2001). However, it is only now that we are beginning to obtain the first insights into the genetic bases of such traits, and these are just partial glimpses of the complex molecular pathways that are likely to be involved (Fisher, in press; Fisher, Lai, \& Monaco, 2003). Recent breakthroughs for gene discovery in neurodevelopmental disorders have benefited from the combined contribution of cognitive science, neuropsychology and genetics, and future unraveling of etiology will also become dependent on other diverse fields, including molecular neuroscience, systems biology and even evolutionary anthropology (Fisher, in press). In coming years, synergy between these disciplines will be critical; it is at the convergence point of such supposedly distinct research areas that the most dramatic and intriguing findings will emerge (Hauser, Chomsky, \& Fitch, 2002). The potential promise of a multidisciplinary perspective for studying neurodevelopmental disorders is 
Fisher: Molecular mechanisms underlying speech and language disorders

perhaps best epitomized by the discovery and subsequent investigations of the FOXP2 gene (Lai, Fisher, Hurst, Vargha-Khadem, \& Monaco, 2001). In the present article, I first outline how (and why) geneticists were able to pinpoint defects in FOXP2 as the cause of one form of inherited speech and language disorder. I then show how, taking this single gene as a focal point, researchers from distinct disciplines are beginning to tease out the relevant pathways for normal and abnormal development, by applying their own areas of expertise. Finally, I hope to persuade the reader that a full understanding of the role of FOXP2 can only come from an integration of findings from multiple levels of analysis. A similar state of affairs will apply to other genes related to speech and language development, which are as yet undiscovered.

\section{THE ART OF GENE HUNTING}

Once it has been established from twin and/or adoption studies that a disorder has a high heritability (i.e., that genetic factors are likely to make a significant contribution to a person's risk of developing impairment), there are a number of ways of tracking down which specific genes may be involved (Botstein \& Risch, 2003). Broadly speaking, the available strategies fall into two categories, "candidate-gene" approaches and "positional" methodologies, although with the recent sequencing of the human genome (Wolfsberg, McEntyre, \& Schuler, 2001), the distinctions between these are beginning to blur. Candidate-gene studies rely on the existence of a prior hypothesis regarding the biological pathways underlying a trait. Researchers then select genes that are already known to be involved in the relevant processes and analyze these in subjects affected with the trait, in the hope that they will discover gene variants (alterations in gene sequence) that are associated with increased risk. The vast majority of studies of attentiondeficit/hyperactivity disorder (ADHD) have exploited this kind of approach, basing the choice of candidate gene on well-established findings relating to efficacy of drug-based therapies. In particular, genes encoding proteins related to dopaminergic systems (most famously, DRD4 and DATl) have been the subject of intense study, given that stimulant medication, primarily methylphenidate, can be effective for treating symptoms of ADHD (DiMaio, Grizenko, \& Joober, 2003). However, for most other neurodevelopmental disorders virtually nothing is known about the potential molecular processes that are involved, making the selection of appropriate candidate genes problematic. The human genome comprises around 24,000-30,000 genes and a large proportion of these, perhaps more than half, are expressed (switched on) in the brain at some point during an organism's life; how does one then choose which gene to analyze? This is where the so-called positional approaches come into their own, because they have proved successful in pinpointing a risk gene without requiring any prior understanding of etiological pathways (Botstein \& Risch, 2003).

At their most simple, positional strategies involve the analysis of inheritance patterns of chromosomal sections as they are passed from generation to generation in a "multiplex" family, that is, a family containing multiple individuals affected with a particular disorder. This kind of endeavor allows a geneticist to locate the chromosomal interval that is most likely to contain the gene that is influencing the 
Fisher: Molecular mechanisms underlying speech and language disorders

disorder under study. Typically the region might span tens or perhaps hundreds of genes (even when pooling findings from many multiplex families), but this nevertheless represents a substantial reduction in the number of potential genes to be studied (compare to the number encoded by the entire genome). Researchers can then focus their efforts on this handful of genes and examine each one in affected subjects, looking for the specific mutation(s) that are contributing to the disorder. As mentioned, there is increasing overlap between positional and candidate gene approaches; it is now common for geneticists to localize a chromosomal region of interest in multiplex families and then prioritize candidate genes from this region based on clues to their likely function. For example, if investigating a chromosomal interval potentially involved in a neurodevelopmental trait, one might prioritize for study a gene previously implicated in neuronal migration or axon growth over one that is known to have a more general "housekeeping" role in many cell types. It is worth bearing in mind, however, that nature will often surprise us; sometimes the most unlikely candidates may turn out to be the true culprits.

At a practical level, how do we localize the risk genes involved in a particular trait or traits, in this case developmental disorders of speech and language? We first recruit families in which multiple individuals display speech and language impairment and take DNA samples from the family members. Then we establish the inheritance patterns of different chromosomal regions as they are passed on through each family, by analyzing the "genotypes" of each sample at a series of naturally occurring DNA variants, known as polymorphic markers. Most of these markers occur in the gaps between the coding parts of genes and can be thought of as signposts marking out particular chromosomal intervals. During transmission of genetic material from one generation to the next, corresponding sections of maternal and paternal chromosome copies are sometimes exchanged (genetic recombination). As a result, the genomic makeup of any individual represents a patchwork of maternal and paternal gene variants from previous generations (Pääbo, 2003). In a typical multigenerational family, genotyping of several hundred DNA markers allows us to reconstruct the inheritance patterns of virtually all chromosomal sections (this process is known as a genome-wide scan or screen). We can then test whether the transmission of any particular chromosomal region is linked to the occurrence of speech and language problems in the family. This is called "linkage mapping," because the existence of recombination allows us to "map" potential risk genes to specific chromosomal locations. Simple statistical tests can be used to indicate whether linkages are likely to represent real findings or have instead arisen due to chance.

The success of carrying out traditional linkage mapping in a multigenerational family depends partly on the number of affected and unaffected individuals, but also on a series of assumptions about the genetic basis of a trait. For example, it assumes the involvement of only one risk gene and requires specification of a particular inheritance model (dominant, recessive, linked to the sex chromosomes, etc.). In general, the majority of neurodevelopmental disorders, including most types of speech and language impairment, violate these crucial assumptions (Fisher \& DeFries, 2002; Fisher et al., 2003; Folstein \& Rosen-Sheidley, 2001). Multiple risk genes could be implicated, modes of inheritance are often obscure, and different genes (with different inheritance patterns) may be involved in 
Fisher: Molecular mechanisms underlying speech and language disorders

different families. This can be a problem, since it is often necessary to pool data from multiple families in order to provide enough power to detect linkage. Moreover, it is difficult to reliably map linkages due to occurrences of incomplete penetrance (individuals with increased genetic risk who remain unaffected) and phenocopies (individuals with low genetic risk who still develop language problems; Lander \& Schork, 1994). This underlying genetic complexity, coupled with difficulties regarding the precise specification of the phenotype (i.e., diagnosis of disorder), has represented a major impediment to progress in mapping genes involved in common neurodevelopmental disorders. New strategies are emerging to handle genetic and phenotypic complexity, but these will not be discussed here. For more detailed accounts of genotyping, genome-wide screens, linkage mapping, and handling complex traits, see recent reviews describing the state of the art in genetic dissection of neurodevelopmental disorders (Fisher \& DeFries, 2002; Fisher et al., 2003; Folstein \& Rosen-Sheidley, 2001).

\section{THE KE FAMILY: BUCKING THE TREND OF GENETIC COMPLEXITY}

In the early 1990s an intriguing case emerged in the literature that appeared to contravene the common observation of genetic complexity in neurodevelopmental disorders (Gopnik \& Crago, 1991; Hurst, Baraitser, Auger, Graham, \& Norell, 1990). This was a large three-generation family, usually referred to as KE, in which 15 individuals suffer from developmental impairments in speech and language acquisition. Remarkably, the inheritance of these problems seemed to suggest involvement of a single copy of one mutated gene, transmitted from generation to generation of the family (i.e., a monogenic dominant pattern of inheritance). Consistent with this, around half of the individuals in the family had escaped from the disorder, presumably because they had not inherited the mutated copy of the putative risk gene. The large number of affected subjects in this family, coupled with the fact that the affected children had grown up in extremely similar environments to their unaffected siblings, represented persuasive (albeit indirect) evidence of a strong and simple genetic effect.

Given the unusual nature of this family, the characterization of their disorder rapidly became both an issue for scientific discussion (Alcock, Passingham, Watkins, \& Vargha-Khadem, 2000; Gopnik \& Crago, 1991; Ullman \& Gopnik, 1999; Vargha-Khadem et al. 1998; Vargha-Khadem, Watkins, Alcock, Fletcher, \& Passingham, 1995; Watkins, Dronkers, \& Vargha-Khadem, 2002) and subject to a certain degree of media speculation (reviewed in Pinker, 2004). The ensuing debate generated extensive comments in various publications, most recently by Fisher et al. (2003) and Marcus and Fisher (2003). Therefore, I will limit myself here to a brief summary of the current consensus (where it exists) regarding the deficits in affected KE members and highlight a few key questions that remain unresolved. The problems inherited in the KE family appear to be restricted to the central nervous system (CNS), an issue I will return to later in the article. One of the most well documented aspects of the disorder is a developmental dyspraxia of the orofacial system; all affected members have difficulty in controlling the complex coordinated mouth movements that underlie speech articulation (Alcock et al., 2000; Vargha-Khadem et al., 1995, 1998; Watkins et al., 2002). Although 
Fisher: Molecular mechanisms underlying speech and language disorders

in some cases the intelligibility of speech can improve over time in response to intensive speech therapy, oromotor deficits remain clearly detectable even in adulthood. As such, tests of orofacial praxis and/or repetition of pronounceable nonsense words (nonwords) represent the most robust methods for diagnosing disorder in the children and adults of the KE family (Watkins et al., 2002). Affected individuals perform normally on basic tests of limb praxis and when making single simple oral movements (Alcock et al., 2000; Watkins et al., 2002). Thus, it is likely that the difficulties reflect deficits in complex motor sequencing. However, it remains unclear whether these deficits are confined to the oromotor system or instead represent a more generalized impairment in motor control that impacts primarily on speech due to the particularly intense demands that human spoken language places on the speech articulators.

The articulation difficulties of affected members are accompanied by deficits in language-related skills, extending to both the expressive and receptive domains and affecting written as well as spoken language (Gopnik \& Crago, 1991; Ullman \& Gopnik, 1999; Vargha-Khadem et al., 1995; Watkins et al., 2002). For example, affected individuals have poor written verbal fluency and have problems spelling nonwords (Watkins et al., 2002). Grammatical abilities are also affected; the disorder impacts on comprehension of complex syntactical structure (evaluated via picture selection), and generation of word inflections or derivations in accordance with grammatical rules (Gopnik \& Crago, 1991; Ullman \& Gopnik, 1999; Watkins et al., 2002). It is worth emphasizing the point that the affected members of the KE family are still able to produce and comprehend language and can communicate with their peers to a certain extent; the disorder appears to involve weakening of language-related abilities across the board, rather than a total absence of any particular linguistic or grammatical skill (Watkins et al., 2002). A remaining area of controversy is the issue of whether the profile of expressive and receptive difficulties observed in the affected members may simply be a secondary result of impaired speech articulation in early development, itself due to a basic motor control deficit (Alcock et al., 2000). Alternatively, it could be hypothesized that the mutation in the KE family has multiple parallel effects on distinct aspects of brain development (this is a common phenomenon in developmental biology, which is known as pleiotropy). In fact, at the neurological and molecular levels, a range of different potential scenarios might account for the relationship between oromotor praxis and linguistic development in the disorder of the KE family (and indeed, in normal speech and language). This question may eventually be resolved through integration of data from different disciplines.

There has been some discussion regarding the nonverbal cognitive abilities of affected subjects in the KE family, fueling a misconception that the disorder is best viewed as a class of general mental retardation. It is indeed accurate to say that the mean performance IQ of affected KE members is significantly lower than that of unaffected subjects in the family (Vargha-Khadem et al., 1995), primarily due to poor performance on only one of the nonverbal subtests used to assess IQ (Watkins et al., 2002), but this is probably a red herring. In the KE family there are unaffected individuals with low nonverbal IQ and affected individuals with normal nonverbal IQ. It is unlikely that the skewed profile of speech and language difficulties observed in affected individuals can be explained 
Fisher: Molecular mechanisms underlying speech and language disorders

by an overall domain-general reduction in cognitive ability. Research into common developmental disorders involving language has shown that the presence of early linguistic problems impacts on other areas later in life, including nonverbal abilities (Rutter \& Mawhood, 1991; Tallal, Townsend, Curtiss, \& Wulfeck, 1991).

\section{FROM DISORDER TO GENE AND BACK AGAIN}

Despite the complexities of the cognitive profile of the disorder segregating in the KE family, the inheritance pattern strongly suggested a simple genetic basis. Using traditional linkage mapping with DNA markers of the kind I described earlier in the article, we were able to formally prove this, and localize the putative defective gene to a small interval on one section of chromosome 7 (Fisher, VarghaKhadem, Watkins, Monaco, \& Pembrey, 1998). Due to the availability of human genomic sequence data in online databases, we could determine that there were around 70 genes in this genomic region (Lai et al., 2000), and so began to screen a subset of these in search of a mutation. These efforts were cut short by the discovery of a child (unrelated to the KE family) who had a similar diagnosis of severe verbal dyspraxia accompanied by substantial impairment of expressive and receptive language (Lai et al., 2000). In this case the disorder was associated with a gross chromosomal rearrangement (of a type known as a translocation) in which a section of chromosome 5 had been exchanged with a section of chromosome 7 . Intriguingly, the point of breakage (the "breakpoint") on chromosome 7 was localized to the same interval that had been implicated in the KE family, and so we hypothesized that disruption of the same gene was responsible in both cases. Precise mapping of the breakpoint in the child with the translocation indicated that it was directly interrupting a novel gene that had not, at the time of discovery, been fully characterized (Lai et al., 2001). A mutation search of this same gene in the KE family then revealed a single tiny alteration to its coding sequence, found in every affected individual. In all the unaffected family members (plus a large panel of unrelated control subjects), the coding sequence of the gene was normal (Lai et al., 2001).

How could a minor change in one letter of DNA within this novel gene be responsible for such a major alteration in brain function in the affected KE individuals? In order to answer this question we took the DNA sequence of the gene and predicted the resulting amino acid sequence of the protein that it was likely to encode (this is a standard code-cracking procedure; invariant rules describe how consecutive triplets of nucleotide letters get translated into strings of amino acids during protein assembly in the cell). We then compared the amino acid sequence to databases of current known proteins that had already been studied. This comparison identified a small stretch of sequence (called a "forkhead-box" or FOX domain) in the predicted protein, found in very similar form in a group of over 40 other human proteins (Carlsson \& Mahlapuu, 2002). Mammalian forkhead proteins are clustered into related subgroups, depending on the degree of similarity between their forkhead domains, and each subgroup is arbitrarily assigned with a different letter of the alphabet (Kaestner, Knöchel, \& Martinez, 2000). The protein encoded by the novel gene we had identified was given the official symbol FOXP2, 
Fisher: Molecular mechanisms underlying speech and language disorders

because it was the second member to be discovered in the P subgroup of FOX proteins.

The presence of the forkhead domain gave the first pointers to both the function of the FOXP2 protein and the significance of the gene mutation in the KE family (Lai et al., 2001). The forkhead box is an 80-100 amino acid domain whose three-dimensional structure allows it to bind to DNA. Intensive studies of forkhead proteins in a variety of species from fungi to mice have reliably established that they act as transcription factors, helping to regulate the abundance of other proteins in the cell (Carlsson \& Mahlapuu, 2002). They do this by binding to stretches of DNA found upstream of the coding parts of genes, and then are able to influence the amount of transcription of the gene in question. (During transcription the information encoded in the chromosomal DNA of a particular gene is converted into an intermediate messenger molecule, made of RNA. The message is further processed and then passed on to the protein building machinery of the cell where it acts as a template for making the encoded protein. The quantity of messenger molecules is a major factor determining the abundance of resulting protein, although there are other points of regulation in the system. Thus, regulation of transcription is a powerful tool for modifying protein levels.) A key aspect of forkhead protein function is that they do not act indiscriminately on all genes. Instead each different forkhead protein is able to regulate transcription of only a subset of genes, known as downstream targets. The specificity of any particular forkhead protein is partly determined by the structure of its forkheadbox domain, leading it to bind more strongly to some DNA sequences than others (Carlsson \& Mahlapuu, 2002). The choice of targets influenced by a forkhead protein may also vary depending on the cellular environment (due to interactions between forkhead proteins and other regulatory cofactors) and could involve activation (increased transcription) or repression (reduced transcription) of gene expression.

Given their function in controlling profiles of gene expression in the cell, forkhead proteins (like other transcription factors) can have diverse roles in multiple biological pathways. Many of the known forkhead proteins are critical regulators of embryological development, involved in the sequence of ontogenetic events that turn a small bundle of undifferentiated cells into a complex and highly organized fetus with manifold specialized tissues and organs (Carlsson \& Mahlapuu, 2002). As such, mutation of different forkhead genes has been linked to a range of developmental disorders in both humans and mice; for example, damage to the FOXC1 gene leads to glaucoma and other eye-related disorders, whereas disruption of FOXP 3 causes a severe form of immune deficiency (Lehmann, Sowden, Carlsson, Jordan, \& Bhattacharya, 2003). For dominantly inherited forkhead-related disorders, where there is one mutant copy and one normal copy of the forkhead gene in question, it is likely that abnormalities result from reduced levels of functional forkhead protein (Lehmann et al., 2003). This idea that forkhead dosage can be critical for normal development is supported by studies of gene disruption in mice. For example, mice have their own version of the FOXCl gene (involved in eye disorders in humans), called Foxc1. If both copies of mouse Foxcl are deleted, so that the mouse cannot make any Foxc1 protein at all, then this is lethal; the mouse dies early on in development (Kume et al., 1998). However, if only one 
copy is deleted, so that the mouse is able to make Foxc1 protein but at reduced levels to normal, then it survives but its eyes do not develop properly (Smith et al., 2000).

This brings us back to FOXP2 and its connection with developmental speech and language disorder. In the case of the child with the translocation, his chromosomal rearrangement directly disrupts one of his copies of FOXP2 (most likely rendering it totally inactive) while leaving the other intact (Lai et al., 2001). Therefore, his disorder appears to be associated with reduced dosage of functional $F O X P 2$. In the KE family, there is a different type of gene disruption, a mutation of a single nucleotide, but again this affects only one copy of FOXP2 in affected subjects. The KE mutation is predicted to change an amino acid that occurs at a critical point in the forkhead domain of the FOXP2 protein, thus interfering with function. In fact, recent biochemical and functional studies of other forkhead proteins has strongly confirmed this prediction, showing that mutation at this position can disrupt cellular localization, DNA-binding activity, and transcriptional regulation of mutant protein (Saleem, Banerjee-Basu, Berry, Baxevanis, \& Walter, 2003). In addition, more and more subjects are now being identified who have speech and language abnormalities associated with hemizygous deletions (i.e., deletions affecting only one chromosomal copy) involving FOXP2 (e.g., Liegeois et al., 2001) although other neighboring genes are also deleted. Based on the clinical data and knowledge of mechanisms of action of forkhead proteins we have hypothesized that reduced amounts of functional FOXP2 protein at a key stage of neurogenesis, and/or in a particular region of the CNS, leads to abnormalities in neural structures that are important for development of speech and language abilities (Lai et al., 2001). Thus, starting simply from the observation of a curious inheritance pattern in a multigenerational pedigree, and using a purely positional approach for gene hunting, it was eventually possible to identify the primary genetic cause, and also propose a plausible molecular mechanism to account for the disorder. However, identification of a mutated gene is not an end point, but rather a beginning, as I will demonstrate in the remainder of the article.

\section{MORE THAN A NAME: FOLLOWING UP FOXP2}

Discovering that disruption of FOXP2 is one cause of developmental speech and language disorder represents hard evidence that alterations to genes are able to influence the acquisition of verbal communication skills. As such, cognitive scientists may be tempted to insert the phrase "the FOXP2 gene" into their favorite hypotheses about the role of a gene or genes in human linguistic prowess, without necessarily modifying their original theories. As I outline below there are compelling reasons to be cautious about doing so. Regardless, FOXP2 is much more than an abstract name to hang a theory on; the gene provides a range of means to answer interesting questions about disease etiology, neurological mechanisms and evolution (Fisher, in press; Marcus \& Fisher, 2003). Some of these questions have already been answered but others are currently the subject of intensive research in my laboratory and elsewhere. 
Fisher: Molecular mechanisms underlying speech and language disorders

\section{Is FOXP2 a gene for speech?}

Despite much speculation to the contrary, FOXP2 cannot accurately be described as a gene for speech. It is true that reduced dosage of functional FOXP2 does impact on vocal communication, but this does not mean that the gene exists in order to endow human beings with the gift of speech and language (Fisher, in press). Mice have their own version of FOXP2, referred to as Foxp2; however (to the best of my knowledge), they are unable to speak. Perhaps mouse Foxp2 is very different from the human version. In fact, this is not the case at all. The mouse and human versions are particularly striking in their similarity; the encoded proteins differ for just 3 amino acids of over 700 (Enard et al., 2002) and are thus likely to have similar functionality. Does the rodent version have anything to do with mouse brain development? There is evidence from examination of expression patterns (where and when the gene itself is switched on and off during development) that mouse Foxp2 and human FOXP2 play corresponding roles in establishing and maintaining neural circuitry (Lai, Gerrelli, Monaco, Fisher, \& Copp, 2003). It is possible that there have been modifications and/or embellishments to the role of the human version in recent human history, as I will elaborate on below; but the point is that the ancestral form of FOXP2 was already vital for CNS development in the common ancestor of humans and rodents.

FOXP2 is not only switched on in the brain, but expression is also seen in defined patterns in the developing lungs, intestinal, and cardiovascular tissue (Shu, Yang, Zhang, Lu, \& Morrisey, 2001). Indeed the mouse version of Foxp2 was initially characterized as an important regulator of lung development (Shu et al., 2001). As for many other transcription factors, FOXP2 is likely to play multiple roles during embryogenesis (and perhaps at other stages of development). The problems of humans with disruption of one copy of FOXP2 appear to be restricted to the $\mathrm{CNS}$, suggesting that a single copy of FOXP2 is enough for the lungs, gut, and heart to develop and function normally. Again, this kind of scenario is commonly observed for other transcription factors (Lehmann et al., 2003). Consequently, FOXP2 must be viewed in the context of systems biology as one element that is recruited for repeated use in multiple differing pathways of some complexity. The CNS specificity of deficits resulting from reduced functional dosage may be explained by differing sensitivity of these distinctive pathways to fluctuations in FOXP2 levels. Is this a reasonable hypothesis? To cite just one comparable example from the literature, mouse Foxcl is expressed in many tissues during embryonic development. As mentioned, a total absence of Foxc1 protein is lethal; mice die due to severe defects in the axial skeleton and cardiovascular system (Kume et al., 1998). However, remember that when only one Foxcl copy is disrupted, a much more restricted pattern of defects occurs, predominantly involving aberrant ocular development (Smith et al., 2000). This illustrates how hard it is to predict consequences of reduced dosage based on expression patterns, because cells of different origins do not necessarily respond in the same way to alterations in protein levels.

Some may suggest that the validity of referring to FOXP2 as a speech gene is simply a question of subjective semantics, and that the simplified view is important to make the research accessible to nonspecialists. However, I have argued that the 
Fisher: Molecular mechanisms underlying speech and language disorders

speech gene view is a damaging caricature of the available data which yields models of the molecular bases of speech and language that are not sufficiently rooted in our current understanding of developmental biology and genetics (Fisher, in press).

\section{Do variants in FOXP2 contribute more widely to common language-related disorders?}

Data from the KE family, the unrelated child with the translocation, and a number of other deletion cases indicate a clear link between loss of FOXP2 function and abnormal speech and language development. An obvious question to ask is how prevalent FOXP2-linked problems may be in the wider population of children with language-related disorders. As noted, these traits are usually complex at the genetic level, and so may potentially involve many different genes (Fisher et al., 2003). Against this background, how much of a contribution does mutation of FOXP2 make? Before answering this question it is worth tackling a subtle but fundamental point that is easily misunderstood by those outside the field of genetics. The mutation observed in the KE family alters the FOXP2 protein at one critical site along its length, leading to disruption of function, but beyond this there is nothing particularly special about the change. The mutation is not a common risk allele that is found in the general population; instead, it is private to the KE family and must have occurred at random in the ancestors of the current family members, most likely in the germ cells of one of the great-grandparents. This was demonstrated by Lai and colleagues in 2001; the KE mutation was never found in screenings of a large number of control individuals, representing nearly 400 independent chromosomal copies (Lai et al., 2001). There is no reason to expect that the KE mutation should be present in other individuals with speech and language disorder, unless they are directly related to the KE family (i.e., cousins of the current living members).

In fact, in affected subjects who are unrelated to the KE family, mutations might have potentially occurred to any of the 2145 nucleotide letters that code for the FOXP2 protein, and many of these possible changes could have altered one of its 715 amino acids in a way that would irrevocably disrupt protein function. For example, the protein contains several important functional domains outside of the forkhead-box motif, including a polyglutamine stretch, a zinc finger, and a leucine zipper; these domains mediate interactions with other proteins involved in transcriptional regulation (Wang, Lin, Li, \& Tucker, 2003). Moreover, some types of mutation can result in "stop" signals being inappropriately placed early on in coding regions of genes, leading to protein truncations. Alterations of this kind can yield truncated products that lack important domains and are thus unable to function, but the mutations may themselves lie some distance from the domains that are being lost. Thus, studies in which language-impaired children from the wider population are tested only for the specific mutation that is private to the $\mathrm{KE}$ family (e.g., "No sign of the FOXP2 mutation"; Meaburn, Dale, Craig, \& Plomin, 2002) are unable to offer insight into the wider impact of FOXP2 gene variants on this disorder. This kind of investigation is analogous to proofreading just a single word at one point of a 2145 -word article and going on to assume that the 
whole article is free from errors. Of course, the accuracy of the particular word that has been checked might be absolutely critical to preserve the meaning of the article (and it may be a word that was previously garbled in an independent print run of the same article), but finding accuracy at this one point does not allow the proofreader to conclude that the rest of the text is correct.

Recent population-based studies have found that FOXP 2 sequence shows particularly low variability in unselected human populations (Enard et al., 2002; Zhang, Webb, \& Podlaha, 2002). In the absence of any common coding variants of FOXP2 (e.g., protein-altering sequence changes that are present at a frequency of $>5 \%$ in unselected populations), one of the best ways to fully explore the contribution of FOXP2 changes to neurodevelopmental disorders is to search for potential mutations along the entire length of its coding sequence, or at least across domains that are known to be functionally important. (In reality, the situation is even more complex than I have implied, because changes outside of the coding region may also impact on function.) Several independent research groups have now screened the entire FOXP 2 coding region in large numbers of cases of specific language impairment and autism (Gauthier et al., 2003; Newbury et al., 2002; Wassink et al., 2002), and it appears unlikely that changes to FOXP2 protein sequence make much of a contribution (if at all) to the incidence of these kinds of language-related disorders. In other studies, Kaminen et al. (2003) found no mutations when investigating the entire $F O X P 2$ coding region in six individuals with developmental dyslexia, whereas O'Brien, Zhang, Nishimura, Tomblin, and Murray (2003) reported similarly negative results in 96 language-impaired subjects, but in this case examined a small region of FOXP2 corresponding to only 40 amino acids of the forkhead-box domain. We are in the process of searching for FOXP2 mutations in subjects with deficits that more closely mimic the problems known to be associated with disruption of this gene. In particular we are focusing on the verbal dyspraxia element of the phenotype, since this seems to be the best diagnostic marker of disorder in the KE family and in the translocation and deletion cases studied thus far. What some have referred to as the FOXP2 mutation (that found in the KE family) is unlikely to be the only point mutation in FOXP2 that exists in the world. Yes, it is the only one to have been reported thus far, but this is partly a historical accident. Regardless of these finer discussion points, it is clear that changes to FOXP2 explain only a tiny fraction of subjects affected with neurodevelopmental disorder. However, it also remains possible that other genes in FOXP2-related pathways may harbor risk variants, so studies of FOXP2 protein function may still be relevant to more common disorders.

\section{What is the function of FOXP2 in the developing brain?}

As a first step toward dissecting the neuromolecular pathways influenced by $F O X P 2$, we and others have analyzed its expression pattern (when and where the gene is transcribed and translated) during fetal brain development (e.g., Ferland, Cherry, Preware, Morrisey \& Walsh, 2003; Lai et al., 2003; Takahashi, Liu, Hirokawa, \& Takahashi, 2003). Concordant with its predicted role as a transcription factor influencing neuronal development, FOXP2 protein is found in the nuclei of neurons (and is absent from glial cells). During early development 
Fisher: Molecular mechanisms underlying speech and language disorders

the rodent and human data are remarkably similar, with complex expression patterns involving a range of brain regions including the cortex, striatum, thalamus, cerebellum, and medulla. (Comparisons at later stages are of restricted scope, because it is not feasible or ethical to run the necessary experiments in later stage human embryos, children, or adults.) Although expression is not neatly limited to any one brain region (as might be suggested by a naïve view of genes related to cognition), the timing and specificity within positive structures still indicate that the gene is subject to precise control (Ferland et al., 2003; Lai et al., 2003; Takahashi et al., 2003). More important, the patterns in some of these regions allow us to begin to form hypotheses about exactly what the gene may be doing in terms of developmental biology. There are a number of key cellular processes that occur during CNS development; typically cells are born and proliferate in particular zones; they may migrate (sometimes over surprisingly large distances and past other cells that have already migrated) to find their final positions; they differentiate into specific types of neurons with their own peculiar profiles of expression of neurotransmitters, receptors, and so forth; they form connections with other cells (via a series of mechanisms such as axon path finding, that I do not discuss here); and some cells are lost via programmed cell death (apoptosis). Each brain structure has its own developmental history, and the relevant pathways have been mapped out in some detail by molecular neuroscientists in recent years (e.g., see reviews by Jain, Armstrong, Barker, \& Rosser, 2001; Wang \& Zoghbi, 2001; Zaki, Quinn, \& Price, 2003). Thus, careful scrutiny of gene expression patterns can give clues as to potential function.

In the cortex, studies of rodents clearly show that Foxp 2 expression is limited to the deeper cortical layers (predominantly layer 6; Ferland et al., 2003; Takahashi et al., 2003). These layers derive from the earliest born cortical progenitor cells, but the timing and pattern of Foxp 2 expression indicate a role that begins after proliferation and migration. Layer 6 neurons predominantly project back to the dorsal thalamus. Mutations of genes like Tbrl and Otxl, which similarly encode transcription factors that are expressed in deeper cortical layers, lead to abnormal formation of back-projections to subcortical regions (Hevner et al., 2001; Weimann et al., 1999). Thus, does Foxp2 have a comparable function in helping to establish corticothalamic connectivity?

Studies of the rat striatum (comprising the caudate and putamen), a site of strong Foxp2 expression, have indicated another intriguing case of sublocalization (Takahashi et al., 2003). The striatum contains two structurally similar but histochemically and functionally distinct compartments, known as the striosomes and the matrix. These express different levels of neurotransmitters, neuropeptides, receptors, and signaling molecules, have distinct neurodevelopmental origins and make different connections to other brain regions (Jain et al., 2001). In rats Foxp2 appears to be much more highly expressed in the striosomal compartment throughout life and may well play a role in differentiation of striosomes (Takahashi et al., 2003). Perhaps (as for the cortex) it regulates some aspect of connectivity. However, it is not yet clear whether the striosomal enrichment of Foxp2 is a general feature that is present in other species.

A final notable instance of precise sublocalization of FOXP2 expression is found in the olivocerebellar system of both rodents and humans. The cerebellum 
is a complex structure made up by a variety of cell types with distinct origins and functions, including Purkinje, granule, Golgi, stellate, and basket cells, as well as deep nuclei (Wang \& Zoghbi, 2001). Of these, FOXP2 is specifically expressed only in deep nuclei and Purkinje cells (Lai et al., 2003). In the medulla, FOXP2 expression is restricted to a structure known as the inferior olive. Climbing fibers from the inferior olivary neurons provide strong synaptic excitation to Purkinje cells (Wang \& Zoghbi, 2001). These fibers organize their topography in an ordered fashion, perhaps in response to polarity cues from Purkinje cells. Consequently, coincident expression in the different neurons within these circuits suggests a role for FOXP2 in establishing olivocerebellar connectivity.

Overall, based on these developmental expression patterns, one might make an educated guess that FOXP2 is involved in the connectivity of corticostriatal and olivocerebellar circuitry and that this role is evolutionarily conserved; that is, it was already important in the common ancestor of humans and rodents. However, there is a limit to the scope of these kinds of experiments. In the near future, it will be possible to rigorously evaluate the connectivity hypothesis by studying the course of brain development in mice in which the Foxp2 gene has been artificially disrupted. Of course, it is possible that Foxp2 may do different things in different brain regions or at alternative timepoints, but even these more complex scenarios will be amenable to transgenic mouse technology, which has become extremely sophisticated in recent years. It is intriguing to note that anatomical and functional neuroimaging of humans with FOXP2 mutation has highlighted abnormalities in multiple regions concordant with sites of gene expression, including the striatum (most significantly the caudate) and cerebellum, as well as Broca's area in the cortex (e.g., Belton, Salmond, Watkins, Vargha-Khadem, \& Gadian, 2003; Liegeois et al., 2003; Vargha-Khadem et al., 1998).

\section{What is the evolutionary history of FOXP2 in humans and other primates?}

I have suggested that FOXP2 may play an evolutionarily conserved role (or roles) in the corticostriatal and olivocerebellar circuits of human and mouse brains, based on dramatic similarities between protein sequences and developmental expression patterns in these different species. However, the data from subjects with disrupted FOXP2 indicate that the gene is involved in neural circuitry underlying speech and language (although this is not its only function), and these are abilities that are unique to humans. How can all these findings be resolved? Clues have come from comparisons between humans and our closest living relatives, chimpanzees. Remember that there are three amino acid substitutions that distinguish the human and mouse versions of FOXP2 protein. It turns out that one of these coding changes must have occurred on the lineage that led to the mouse, at some point in the last 70 million years (Enard et al., 2002; Zhang et al., 2002). By contrast the other two coding changes arose uniquely on the human lineage, after the split from the chimpanzee lineage, in the last 5-6 million years. In other words, despite the striking similarities between all mammalian versions of FOXP2, there appears to have been accelerated evolution of the protein sequence in recent history, specifically on the lineage that led to modern humans. Two independent studies analyzed the diversity of genomic DNA surrounding the human-specific changes 
Fisher: Molecular mechanisms underlying speech and language disorders

of FOXP2 in a series of human populations with distinctive origins (Enard et al., 2002; Zhang et al., 2002). They concluded that these changes had been the subject of positive selection in recent human history, most likely within the last 200,000 years (although the error bars on this estimate are rather large and it relies on various assumptions about population genetics). Curiously, the appearance and rapid fixation of the two human-specific FOXP2 changes seems to have coincided with the estimated time of emergence of spoken language in human populations (Boyd \& Silk, 2000).

Does this mean that FOXP2, or at least the version that is found in modern humans, is a speech gene after all? Were the modifications of the gene in recent human history the major factor that drove the evolution of human vocal communication? As before, we need to be cautious about interpreting these data and take care to consider the full context of FOXP2 findings when constructing hypotheses about the role of the gene in evolution. First, it is important to be aware that FOXP2 is not the only gene to have undergone positive selection in human history (although it may be one of the most interesting at present given its involvement in speech and language). For example, using large-scale sequence analyses Clark et al. (2003) identified hundreds of genes that show significant evidence of positive selection on the human lineage following the split from the chimpanzees, although more precise dating of these effects has not yet been attempted. Second, it is not currently known how the human-specific changes in FOXP2 might impact on protein function. Although we previously suggested that one of the changes might create a potential new target site for regulation of the FOXP2 protein by other proteins (Enard et al., 2002), this was a tentative prediction based on sequence information. Moreover, it is not possible that this particular change by itself drove the appearance of speech and language, because an equivalent change has arisen independently in carnivores (Zhang et al., 2002). In fact both the human-specific changes lie in a region of the FOXP2 protein that is currently poorly understood at a functional level. Although we cannot go back in time to discover exactly why the human-specific changes spread through the population, we can perform functional experiments using cell lines to compare the human and chimp proteins and thus hope to gain valuable insight into the biological impact of the changes.

Rather than invoking selection of the modern human version of FOXP2 as the major force that produced a population of chatty humans, it seems more reasonable to place evolution of this gene modestly within a framework of other events that contributed on our pathway toward linguistic prowess. Integration of data from different disciplines could again be a key to unlock this issue. I have discussed above how molecular neuroscience and developmental biology hint at a conserved role for mammalian FOXP2 in establishing connectivity of corticostriatal and olivocerebellar circuits. Such circuits are known to underlie control of complex motor activities (Middleton \& Strick, 2000; Welsh, Lang, Suglhara, \& Llinas, 1995). Perhaps, at a time when people were already beginning to use more complex vocalizations (for reasons unrelated to FOXP2), a random alteration to FOXP2 (e.g., one of the human-specific amino acid changes) yielded subtle modifications in connectivity in FOXP2-expressing circuitry in the first humans harboring the change. In other words, this would have involved modification of existing mechanisms rather than emergence of novel ones. Individuals with altered 
FOXP2 may have had a selective advantage in vocal communication as a consequence of improved skill in control of the speech articulators resulting from the altered architecture of motor circuits. Such an advantage could have led to rapid spreading of the new form of FOXP2 through the population, assuming that vocal communication was already becoming an important feature of human behavior. In this view, FOXP2 change was just one of a number of genetic and environmental alterations that contributed to speech evolution, and given the interdependence of elements in developmental systems, it is possible that interaction between these different (as yet unknown) factors was also critical. Even if such a multifactorial model of FOXP2 evolution is accurate (and it remains speculation at this stage), many questions remain unanswered. For example, the hypothesis focuses on the role of FOXP2 in complex co-ordination, mediated by its influence on circuitry related to motor control, thus placing the emphasis on speech articulation and sidestepping thorny issues of how exactly this may relate to language itself. However, it is becoming clear that the corticostriatal and olivocerebellar circuits are not only involved in motor function, but are also important in aspects of human higher cognition (Middleton \& Strick, 2000). Most important, this model is intentionally limited to discussing the role of FOXP2, because that is the only case where there is enough molecular and developmental data to speak to the issue. With the rapid accumulation of further empirical data from humans, chimpanzees, and other species (e.g., Clark et al., 2003), it will be possible in the near future to directly assess the significance of FOXP2 in human evolution in relation to the effects of other genes that have undergone recent positive selection. Thus, the FOXP2 findings are just a taste of things to come.

\section{CONCLUSION}

To my mind, the most exciting aspects of the FOXP2 story are not the answers that have been provided (or hinted at) thus far, but rather the new questions that we are now able to ask, using this gene as a starting point. Scientists are adopting a wide variety of approaches to tease apart the role (or roles) of FOXP2 at different levels; determining the physical structure of the protein; identifying downstream target genes and interacting cofactors; investigating its effects on neuronal cells in culture; asking how it influences patterning and performance of the central nervous system in model organisms; integrating findings with neuroimaging data from humans with mutations; and so on. Moreover, it is likely that other genes will be discovered in the coming years that also play significant roles in speech and language development, and these will be amenable to similar treatment. Thus, this research area, perhaps more than any other, shows how molecular genetics can bridge seemingly distant fields from linguistics, psychology, and neuroscience to developmental biology and evolutionary anthropology. Finally, for those who expected that FOXP2 would provide a simple neurological explanation for the associated speech and language disorder (e.g., that the gene directs formation of just one brain region) or a straightforward answer to the mysteries of human evolution (e.g., that this could be a human-specific speech gene), this research continues to present many fascinating surprises. 
Fisher: Molecular mechanisms underlying speech and language disorders

\section{ACKNOWLEDGMENTS}

Dr. Fisher is a Royal Society Research Fellow. His research is also funded by project grants from the Wellcome Trust and the Medical Research Council.

\section{REFERENCES}

Alcock, K. J., Passingham, R. E., Watkins, K. E., \& Vargha-Khadem, F. (2000). Oral dyspraxia in inherited speech and language impairment and acquired dysphasia. Brain and Language, 75, $17-33$.

Belton, E., Salmond, C. H., Watkins, K. E., Vargha-Khadem, F., \& Gadian, D. G. (2003). Bilateral brain abnormalities associated with dominantly inherited verbal and orofacial dyspraxia. Human Brain Mapping, 18, 194-200.

Bishop, D. V. M. (2001). Genetic and environmental risks for specific language impairment in children. Philosophical Transactions of the Royal Society of London B Biological Sciences, 356, 369380.

Botstein, D., \& Risch, N. (2003). Discovering genotypes underlying human phenotypes: Past successes for mendelian disease, future approaches for complex disease. Nature Genetics, 33(Suppl.), 228-237.

Boyd, R., \& Silk, J. B. (2000). How humans evolved. New York: W. W. Norton.

Carlsson, P., \& Mahlapuu, M. (2002). Forkhead transcription factors: Key players in development and metabolism. Developmental Biology, 250, 1-23.

Clark, A. G., Glanowski, S., Nielsen, R., Thomas, P. D., Kejariwal, A., Todd, M. A., Tanenbaum, D. M., Civello, D., Lu, F., Murphy, B., Ferriera, S., Wang, G., Zheng, X., White, T. J., Sninsky, J. J., Adams, M. D., \& Cargill, M. (2003). Inferring nonneutral evolution from human-chimpmouse orthologous gene trios. Science, 302, 1960-1963.

DiMaio, S., Grizenko, N., \& Joober, R. (2003). Dopamine genes and attention-deficit hyperactivity disorder: A review. Journal of Psychiatry and Neuroscience, 28, 27-38.

Enard, W., Przeworski, M., Fisher, S. E., Lai, C. S. L., Wiebe, V., Kitano, T., Monaco, A. P., \& Pääbo, S. (2002). Molecular evolution of FOXP2, a gene involved in speech and language. Nature, $418,869-872$.

Ferland, R. J., Cherry, T. J., Preware, P. O., Morrisey, E. E., \& Walsh, C. A. (2003). Characterization of Foxp2 and Foxp1 mRNA and protein in the developing and mature brain. Journal of Comparative Neurology, 460, 266-279.

Fisher, S. E. (in press). Tangled webs: Tracing the connections between genes and cognition. Cognition.

Fisher, S. E., \& DeFries, J. C. (2002). Developmental dyslexia: Genetic dissection of a complex cognitive trait. Nature Reviews Neuroscience, 3, 767-780.

Fisher, S. E., Lai, C. S. L., \& Monaco, A. P. (2003). Deciphering the genetic basis of speech and language disorders. Annual Review of Neuroscience, 26, 57-80.

Fisher, S. E., Vargha-Khadem, F., Watkins, K. E., Monaco, A. P., \& Pembrey, M. E. (1998). Localisation of a gene implicated in a severe speech and language disorder. Nature Genetics, 18, 168170 .

Folstein, S. E., \& Rosen-Sheidley, B. (2001). Genetics of autism: Complex aetiology for a heterogeneous disorder. Nature Reviews Genetics, 2, 943-955.

Gauthier, J., Joober, R., Mottron, L., Laurent, S., Fuchs, M., De Kimpe, V., \& Rouleau, G. A. (2003) Mutation screening of FOXP2 in individuals diagnosed with autistic disorder. American Journal of Medical Genetics, 118A, 172-175.

Gopnik, M., \& Crago, M. B. (1991). Familial aggregation of a developmental language disorder. Cognition, 39, 1-50.

Hauser, M. D., Chomsky, N., \& Fitch, W. T. (2002). The faculty of language: What is it, who has it, and how did it evolve? Science, 298, 1569-1579.

Hevner, R. F., Shi, L., Justice, N., Hsueh, Y., Sheng, M., Smiga, S., Bulfone, A., Goffinet, A. M., Campagnoni, A. T., \& Rubenstein, J. L. (2001). Tbr1 regulates differentiation of the preplate and layer 6. Neuron, 29, 353-366.

Hurst, J. A., Baraitser, M., Auger, E., Graham, F., \& Norell, S. (1990). An extended family with a dominantly inherited speech disorder. Developmental Medicine and Child Neurology, 32, $347-355$. 
Fisher: Molecular mechanisms underlying speech and language disorders

Jain, M., Armstrong, R. J., Barker, R. A., \& Rosser, A. E. (2001). Cellular and molecular aspects of striatal development. Brain Research Bulletin, 55, 533-540.

Kaestner, K. H., Knöchel, W., \& Martinez, D. E. (2000). Unified nomenclature for the winged helix/forkhead transcription factors. Genes and Development, 14, 142-146.

Kaminen, N., Hannula-Jouppi, K., Kestila, M., Lahermo, P., Muller, K., Kaaranen, M., Myllyluoma, B., Voutilainen, A., Lyytinen, H., Nopola-Hemmi, J., \& Kere J. (2003). A genome scan for developmental dyslexia confirms linkage to chromosome 2 p11 and suggests a new locus on 7q32. Journal of Medical Genetics, 40, 340-345.

Kume, T., Deng, K. Y., Winfrey, V., Gould, D. B., Walter, M. A., \& Hogan, B. L. (1998). The forkhead/winged helix gene $M f l$ is disrupted in the pleiotropic mouse mutation congenital hydrocephalus. Cell, 93, 985-996.

Lai, C. S. L., Fisher, S. E., Hurst, J. A., Levy, E. R., Hodgson, S., Fox, M., Jeremiah, S., Povey, S., Jamison, D. C., Green, E. D., Vargha-Khadem, F., \& Monaco, A.P. (2000). The SPCH1 region on human 7q31: Genomic characterization of the critical interval and localization of translocations associated with speech and language disorder. American Journal of Human Genetics, 67, 357-368.

Lai, C. S. L., Fisher, S. E., Hurst, J. A., Vargha-Khadem, F., \& Monaco, A. P. (2001). A novel forkheaddomain gene is mutated in a severe speech and language disorder. Nature, 413, 519-523.

Lai, C. S. L., Gerrelli, D., Monaco, A. P., Fisher, S. E., \& Copp, A. J. (2003). FOXP2 expression during brain development coincides with adult sites of pathology in a severe speech and language disorder. Brain, 126, 2455-2462.

Lander, E. S., \& Schork, N. J. (1994). Genetic dissection of complex traits. Science, 265, 20372048.

Lehmann, O. J., Sowden, J. C., Carlsson, P., Jordan, T., \& Bhattacharya, S. S. (2003). Fox's in development and disease. Trends in Genetics, 19, 339-344.

Liegeois, F., Baldeweg, T., Connelly, A., Gadian, D. G., Mishkin, M., \& Vargha-Khadem, F. (2003). Language fMRI abnormalities associated with FOXP2 gene mutation. Nature Neuroscience, 6 , 1230-1237.

Liegeois, F. J., Lai, C. S. L., Baldeweg, T., Fisher, S. E., Monaco, A. P., Connelly, A., \& VarghaKhadem, F. (2001). Behavioural and neuroimaging correlates of a chromosome 7q31 deletion containing the SPCH1 gene. Society of Neuroscience Abstracts, 27, Program No. 529.17.

Marcus, G. F., \& Fisher, S. E. (2003). FOXP2 in focus: What can genes tell us about speech and language? Trends in Cognitive Sciences, 7, 257-262.

Meaburn, E., Dale, P. S., Craig, I. W., \& Plomin, R. (2002). Language-impaired children: No sign of the FOXP2 mutation. Neuroreport, 13, 1075-1077.

Middleton, F. A., \& Strick, P. L. (2000). Basal ganglia and cerebellar loops: Motor and cognitive circuits. Brain Research Reviews, 31, 236-250.

Newbury, D. F., Bonora, E., Lamb, J. A., Fisher, S. E., Lai, C. S. L., Baird, G., Jannoun, L., Slonims, V., Stott, C. M., Merricks, M. J., Bolton, P. F., Bailey, A., Monaco, A. P., \& The International Molecular Genetic Study of Autism Consortium. (2002). FOXP2 is not a major susceptibility gene for autism or specific language impairment (SLI). American Journal of Human Genetics, 70, 1318-1327.

O’Brien, E. K., Zhang, X., Nishimura, C., Tomblin, J. B., \& Murray, J. C. (2003). Association of specific language impairment (SLI) to the region of 7q31. American Journal of Human Genetics, 72, 1536-1543.

Pääbo, S. (2003). The mosaic that is our genome. Nature, 421, 409-412.

Pinker, S. (1994). The language instinct. London: Allen Lane.

Rutter, M., \& Mawhood, L. (1991). The long-term psychosocial sequelae of specific developmental disorders of speech and language. In M. Rutter \& P. Casaer (Eds.), Biological risk factors for psychosocial disorders (pp. 233-259). Cambridge: Cambridge University Press.

Saleem, R. A., Banerjee-Basu, S., Berry, F. B., Baxevanis, A. D., \& Walter, M. A. (2003). Structural and functional analyses of disease-causing missense mutations in the forkhead domain of FOXC1. Human Molecular Genetics, 12, 2993-3005.

Shu, W., Yang, H., Zhang, L., Lu, M. M., \& Morrisey, E. E. (2001). Characterization of a new subfamily of winged-helix/forkhead (Fox) genes that are expressed in the lung and act as transcriptional repressors. Journal of Biological Chemistry, 276, 27488-27497.

Smith, R. S., Zabaleta, A., Kume, T., Savinova, O. V., Kidson, S. H., Martin, J. E., Nishimura, D. Y., Alward, W. L., Hogan, B. L., \& John, S. W. (2000). Haploinsufficiency of the transcription 
Fisher: Molecular mechanisms underlying speech and language disorders

factors FOXC1 and FOXC2 results in aberrant ocular development. Human Molecular Genetics, 9, 1021-1032.

Takahashi, K., Liu, F. C., Hirokawa, K., \& Takahashi, H. (2003). Expression of Foxp2, a gene involved in speech and language, in the developing and adult striatum. Journal of Neuroscience Research, $73,61-72$.

Tallal, P., Townsend, J., Curtiss, S., \& Wulfeck, B. (1991). Phenotypic profiles of language-impaired children based on genetic/family history. Brain and Language, 41, 81-95.

Ullman, M. T., \& Gopnik, M. (1999). Inflectional morphology in a family with inherited specific language impairment. Applied Psycholinguistics, 20, 51-117.

Vargha-Khadem, F., Watkins, K., Alcock, K., Fletcher, P., \& Passingham, R. (1995). Praxic and nonverbal cognitive deficits in a large family with a genetically transmitted speech and language disorder. Proceedings of the National Academy of Sciences USA, 92, 930-933.

Vargha-Khadem, F., Watkins, K. E., Price, C. J., Ashburner, J., Alcock, K. J., Connelly, A., Frackowiak, R. S., Friston, K. J., Pembrey, M. E., Mishkin, M., Gadian, D. G., \& Passingham, R. E. (1998). Neural basis of an inherited speech and language disorder. Proceedings of the National Academy of Sciences USA, 95, 12695-12700.

Wang, B., Lin, D., Li, C., \& Tucker, P. (2003). Multiple domains define the expression and regulatory properties of Foxp1 forkhead transcriptional repressors. Journal of Biological Chemistry, 278, 24259-24268.

Wang, V. Y., \& Zoghbi, H. Y. (2001). Genetic regulation of cerebellar development. Nature Reviews Neuroscience, 2, 484-491.

Wassink, T. H., Piven, J., Vieland, V. J., Pietila, J., Goedken, R. J., Folstein, S. E., \& Sheffield, V. C. (2002). Evaluation of FOXP2 as an autism susceptibility gene. American Journal of Medical Genetics, 114, 566-569.

Watkins, K. E, Dronkers, N. F., \& Vargha-Khadem, F. (2002). Behavioural analysis of an inherited speech and language disorder: Comparison with acquired aphasia. Brain, 125, 452-464.

Weimann, J. M., Zhang, Y. A., Levin, M. E., Devine, W. P., Brulet, P., \& McConnell, S. K. (1999). Cortical neurons require Otx 1 for the refinement of exuberant axonal projections to subcortical targets. Neuron, 24, 819-831.

Welsh, J. P., Lang, E. J., Suglhara, I., \& Llinas, R. (1995). Dynamic organization of motor control within the olivocerebellar system. Nature, 374, 453-457.

Wolfsberg, T. G., McEntyre, J., \& Schuler, G. D. (2001). Guide to the draft human genome. Nature, 409, 824-826.

Zaki, P. A., Quinn, J. C., \& Price, D. J. (2003). Mouse models of telencephalic development. Current Opinion in Genetics and Development, 13, 423-437.

Zhang, J., Webb, D. M., \& Podlaha O. (2002). Accelerated protein evolution and origins of humanspecific features: Foxp2 as an example. Genetics, 162, 1825-1835. 